\title{
Evaluation of Bacteriological Profile of Post Operative Wound Infections in Surgical Wards
}

\author{
Neha Goel $^{1}$, Jaya T. Hemnani ${ }^{2} *$ and Parnika Garg ${ }^{3}$ \\ ${ }^{1}$ Department of Microbiology, ${ }^{2}$ Department of General Surgery, ${ }^{3}$ Department of Pathology, \\ GS Medical College, Pilkhuwa, Dist. Hapur (UP) - 245 304, India \\ *Corresponding author
}

A B S T R A C T

Surgical site infection is one of the most common causes of healthcare associated infections. The present study was conducted to evaluate bacteriological profile of

Keywords

Staphylococcus aureus,

Klebsiella pneumoniae, Wound

Article Info

Accepted:

25 January 2020

Available Online:

10 February 2020 post operative wound infections in surgical wards. The present study was conducted on 115 patients admitted in surgical wards (orthopedic, general surgery, ophthalmology, obstetrics and gynecology and otorhinolaryngology). Two sterile cotton swabs were collected aseptically from each patient suspected of having SSI. Out of 115 patients, $52(45.2 \%)$ cases were found to be positive. Common isolates were Staphylococcus aureus in $31 \%$, Klebsiella pneumoniae in 26\%, Pseudomonas aeruginosa in 17\%, Escherichia coli in $16 \%$ and Acinetobacter spp in $10 \%$. The difference was significant $(\mathrm{P}<0.05)$.We found that $K$. pneumoniae and $P$. aeruginosa strains showed high degree of resistance for ampicillin (100\%). Gram negative isolates showed even higher rate of resistance and ciprofloxacin were found resistant for most of the gram negative isolates. Methicillin resistance was seen in $20.4 \%$ of all the S. aureus isolates. Authors found that most common bacteria isolate in surgical wounds was Staphylococcus aureus, Klebsiella pneumonia, Pseudomonas aeruginosa and Escherichia coli.

\section{Introduction}

Surgical site infections (SSI), one of the most common causes of healthcare associated infections are a common complication associated with surgery, with a reported incidence rates of 2-20\%. Surgery has made great advances in last 3 quarters of this century and postoperative wound infection is the most common complication faced by surgeon since the advent of surgery. ${ }^{1}$

The centre for Disease Control has defined surgical site infections (SSIs) as those infections occurring within 30 days of surgery. Wound infections including SSIs are among the leading nosocomial infections with alarming morbidity and mortality rates the world over. This is in spite of massive campaigns on strict adherence to standard 
surgical and dressing procedures. In the United Kingdom, surgical site infections alone account for seventy seven percent of deaths of surgical patients. ${ }^{2}$

Microorganisms invade and colonize surgical wounds, burns, bruises, cuts and open sores in the event of any breach in septic procedures. ${ }^{3}$ The organisms are either of endogenous or exogenous origin: exogenous when natural pathogens from environment colonize and multiply in the wounds and endogenous when resident flora infiltrate from their natural habitats into these wounds. ${ }^{4}$ Although microbes could contaminate surgical wounds, not all become infected; this is because innate host defenses are quite efficient in the elimination of contaminants. A wide range of organisms are known to infect wounds, they span from gram positive cocci and bacilli, gram negative cocci and bacilli, spore-formers and non-spore formers, aerobes and anaerobes with Staphylococcus aureus leading the pack in frequency of occurrence. ${ }^{5}$ The present study was conducted to evaluate bacteriological profile of post operative wound infections in surgical wards.

\section{Materials and Methods}

The present study was conducted in the department of Microbiology. It comprised of 115 patients admitted to surgical wards such as orthopedic, general surgery, ophthalmology, obstetrics and gynecology and otorhinolaryngology. Inclusion criteria was patients of both genders above 14 years of age, who had surgical wound pus discharge, with serous or seropurulent discharge and with signs of sepsis present concurrently. Patients who had wounds with cellulitis and no drainage were excluded from the study. All patients were informed regarding the study and written consent was obtained. Ethical approval was obtained from institutional ethical committee prior to the study.
Two sterile cotton swabs were collected aseptically from each patient suspected of having SSI. Gram stained preparation was made. The other swab was inoculated on blood agar and Mac Conkey agar and incubated at $37^{\circ} \mathrm{C}$ for 48 hours before being reported as sterile. Growth on culture plate was identified by its colony characters \& the battery of standard biochemical tests.

Antimicrobial sensitivity testing was carried out by Kirby-Baeur disk diffusion method on Mueller Hinton agar as per CLSI guidelines. Methicillin resistance in Staphylococcus isolates was tested by cefoxitin disc diffusion method. Extended spectrum beta lactamases (ESBL) production were detected. Results thus obtained were subjected to statistical analysis. $\mathrm{P}$ value less than 0.05 was considered significant.

\section{Results and Discussion}

Table I shows that out of 115 patients, 52 $(45.2 \%)$ cases were found to be positive. Table II, graph I shows that common isolates were Staphylococcus aureus in $43 \%$, Klebsiella pneumoniae in 14\%, Pseudomonas aeruginosa in 17\%, Escherichia coli in $16 \%$ and Acinetobacter spp in 10\%. The difference was significant $(\mathrm{P}<0.05)$.

Table III shows that $K$. pneumoniae and $P$. aeruginosa strains showed high degree of resistance for ampicillin (100\%). Gram negative isolates showed even higher rate of resistance and ciprofloxacin were found resistant for most of the gram negative isolates.

Despite the advances in surgical techniques and better understanding of the pathogenesis of wound infection, management of SSIs remains a significant concern for surgeons and physicians in a health care facility. Hospitals serve as a reservoir for SSIs as they 
harbor a variety of pathogenic microbes. The unrestrained and rapidly spreading resistance to the available array of antimicrobials further contributes to the existing problem. ${ }^{6}$

Risk factors other than microbiological include decreased host resistance, local wound characteristics and operative characteristics have been included. ${ }^{7}$ There is therefore need for assessment of bacteriological status of wounds, published report on the infections and more aggressive campaign on averting the ugly trend in this part of the world. The fact that some swabs yield more than one bacterial isolate suggests that the source of the contamination is external, either due to exposure to dust from surrounding environment or because of the use of non-septic materials in the dressing of surgical sites. The spectrum of bacteria incriminated is the same globally. ${ }^{8}$ The present study was conducted to evaluate bacteriological profile of post operative wound infections in surgical wards.

In present study, out of 115 patients, 52 $(45.2 \%)$ cases were found to be positive. Kaur et $\mathrm{al}^{9}$ found that rate of SSI was $58.00 \%$.

Table.I Distribution of patients

\begin{tabular}{|c|c|c|}
\hline Total & No. of positive cases & $\%$ of positive cases \\
\hline $\mathbf{1 1 5}$ & 52 & $45.2 \%$ \\
\hline
\end{tabular}

Table.II Bacterial isolates obtained from patients

\begin{tabular}{|c|c|c|}
\hline Organism & No. of isolates & \multirow{2}{*}{ P value } \\
\hline Staphylococcus aureus & $43 \%$ & \\
\hline Klebsiella pneumoniae & $14 \%$ & \\
\cline { 1 - 2 } Pseudomonas aeruginosa & $17 \%$ & \\
\hline Escherichia coli & $16 \%$ & \\
\hline Acinetobacter spp & $10 \%$ & \\
\hline
\end{tabular}

Table.III Antibiotic sensitivity pattern of bacterial isolates in surgical site infections

\begin{tabular}{|c|c|c|c|c|c|}
\hline Antibiotics & S. aureus & K. pneumoniae & P. aeruginosa & E. coli & A. Spp \\
\hline AMP & $85.7 \%$ & $100 \%$ & $100 \%$ & $81.4 \%$ & $98 \%$ \\
\hline AMC & $35 \%$ & $100 \%$ & $82 \%$ & $81.4 \%$ & $72 \%$ \\
\hline AMK & $8.4 \%$ & $0 \%$ & $45.6 \%$ & $15.2 \%$ & $16 \%$ \\
\hline Aztreonam & Not tested & $100 \%$ & $74.5 \%$ & $80.4 \%$ & $71.2 \%$ \\
\hline Cefotaxime & Not tested & $65 \%$ & $72.8 \%$ & Not tested & Not tested \\
\hline Ceftazidime & $35.6 \%$ & Not tested & $71.5 \%$ & Not tested & Not tested \\
\hline Ciprofloxacin & $45 \%$ & $34.8 \%$ & $90.6 \%$ & $28.1 \%$ & $26.7 \%$ \\
\hline P value & 0.01 & 0.01 & 0.02 & 0.04 & 0.05 \\
\hline
\end{tabular}


Graph.I Bacterial isolates obtained from patients

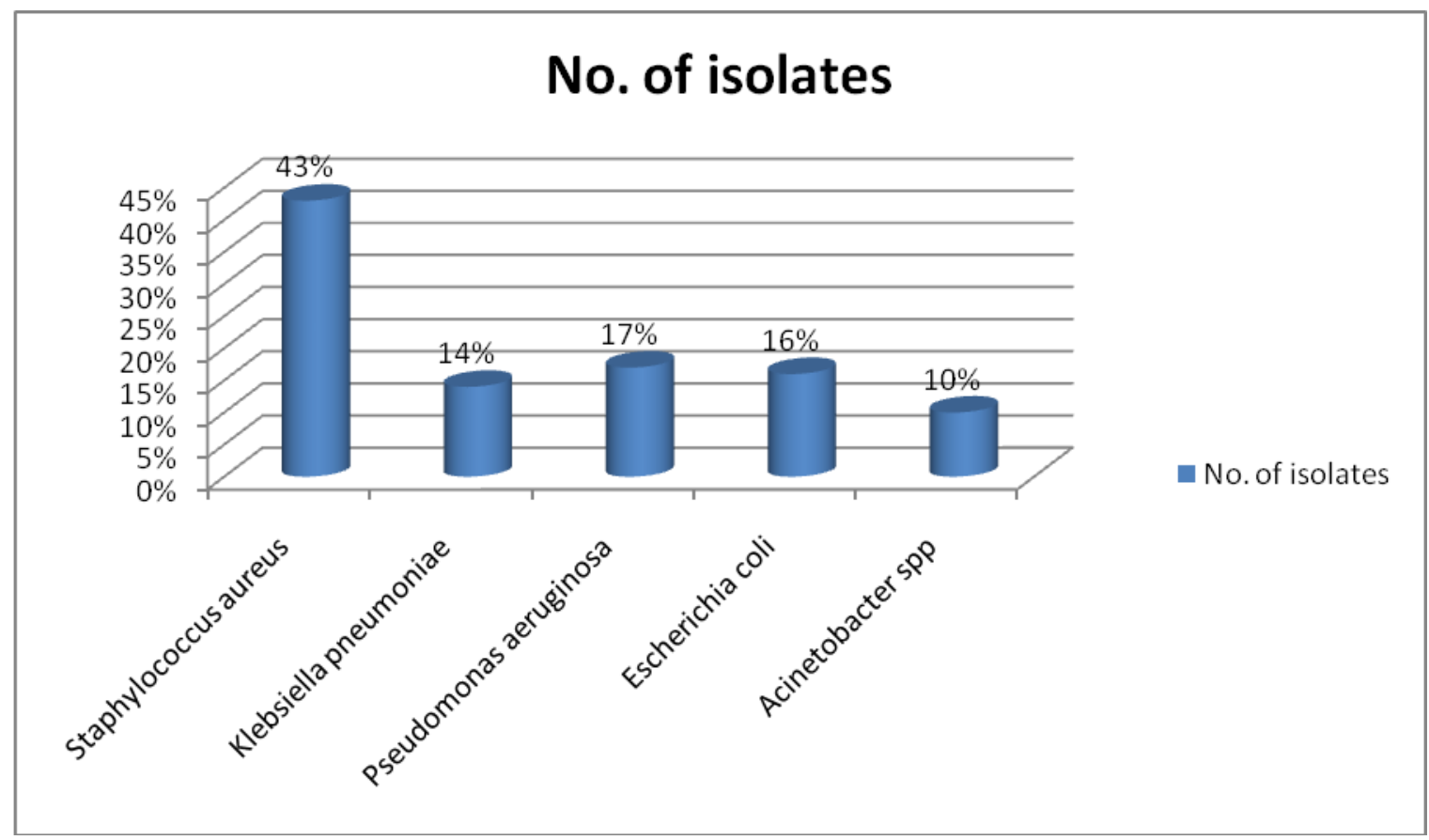

A total of 431 isolates were obtained, out of which $428(99.30 \%)$ were bacterial isolates and $3(0.69 \%)$ were fungal isolates. Monomicrobial growth was seen in 395 $(95.64 \%)$ patients whereas polymicrobial growth was seen in 18 patients $(4.35 \%)$. Out of 428 bacterial isolates, majority of bacteria were gram negative bacteria $(60.74 \%)$ but most common isolate was Staphylococcus aureus. The microbiological profile of the 260 $(60.74 \%)$ gram negative bacteria was Klebsiella pneumoniae followed by Pseudomonas aeruginosa, Escherichia coli, Acinetobacter spp., Citrobacter spp. and Proteus spp. Methicillin resistance was seen in $10.52 \%$ of all the S.aureus isolates and $21.05 \%$ of CONS isolates. No vancomycin resistance in enterococcus was detected. Out of 260 gram negative bacilli, 105 (40.38\%) were ESBL producers.

The present study found that common isolates were Staphylococcus aureus in $31 \%$, Klebsiella pneumoniae in $26 \%$, Pseudomonas aeruginosa in $17 \%$, Escherichia coli in $16 \%$ and Acinetobacter spp in $10 \%$.Chollomet $\mathrm{al}^{10}$ found that two hundred surgical site swabs were collected from two hospitals and investigated. The swabs were cultured and organisms identified according to standard procedures. A prevalence rate of $31.5 \%$ was obtained from the surgical sites investigated. Direct gram staining gave a greater recovery rate of incriminated organisms than cultural methods. Staphylococcus aureus was the most encountered pathogen with $13.0 \%$ prevalence rate followed by Pseudomonas aeruginosa (6.5\%), Proteus mirabilis $(6.0 \%)$ and Klebsiella aerogenes (4.0\%).The least encountered pathogens were Beta-haemolytic streptococci and Escherichia coli with prevalence rates of $1.0 \%$ each. Statistically, chi square analysis showed that there was no significant difference in the number of isolates from the two health facilities at $99 \%$ confidence limit. Susceptibility of isolates was more with the aminoglycosides than with the penicillins. This study has thus revealed that some hospitals are not doing enough to stem the tide of surgical site infections. 
In the present study found that $K$. pneumoniae and $P$. aeruginosa strains showed high degree of resistance for ampicillin (100\%). Gram negative isolates showed even higher rate of resistance and ciprofloxacin were found resistant for most of the gram negative isolates. Methicillin resistance was seen in $20.4 \%$ of all the S.aureus isolates.

In the present study found that most common bacteria isolate in surgical wounds was Staphylococcus aureus, Klebsiella pneumonia, Pseudomonas aeruginosa and Escherichia coli.

\section{References}

1.Pradhan GB, Agrawal J. Comparative study of post-operative wound infection following emergency lower segment caesarean section with and without the topical use of fusidic acid. Nepal Med Coll J., 2009; 11(3): 189-91.

2.Mahmood A. Bacteriology of surgical site infections and antibiotic susceptibility pattern of the isolates at a tertiary care hospital in Karachi. J Pak Med Assoc., 2000; 50: 256-9.

3.Sawhney N, Prabhas R, Singh VA. Post operative wound infections: pattern of bacterial pathogens and their antibiotic sensitivity in a tertiary care hospital of north India. International Journal of Recent Trends in Science and Technology, 2017; 22(3): 214-217.

4.Forbes BA, Sahm DF, Weissfeld AS. Bailey and Scott's Diagnostic Microbiology. 10th ed. St. Louis, Misssouri, USA: Mosby Inc; 1998.

5.Budhani D, Kumar S, Sayal P, Singh S. Bacteriological profile and antibiogram of surgical site infection/ post-operative wound infection. International Journal of Medical Research and Review. 2016; 4(11): 1994-1999

6.Negi V, Pal S, Juyal D, Sharma MK, Sharma N. Bacteriological Profile of Surgical Site Infections and Their Antibiogram: A Study From Resource Constrained Rural Setting of Uttarakhand State, India. J ClinDiagn Res., 2015; 9(10): 17-20.

7.Mundhada AS, Tenpe S. A study of organisms causing surgical site infections and their antimicrobial susceptibility in a tertiary care government hospital. Indian J PatholMicrobiol. 2015; 58: 195-200.

8.Saleem M. Bacterial Profile and Antimicrobial Susceptibility. Pattern of Surgical Site Infections - A Retrospective Study. Indian Journal of Applied Research, 2015; 5(10): 204206.

9.Kaur K, Loveena Oberoi, Pushpa Devi. Bacteriological profile of surgical site infections. IAIM 2017; 4(12): 77- 83.

10.Chollom SC, Agada GO, Gotep JG, Gbise DS, Mwankon SE, Okwori AJ Bacteriological profile of infected surgical sites in Jos, Nigeria. Proteus. 2012 Jan 1;3(10):13.

\section{How to cite this article:}

Neha Goel, Jaya T. Hemnani and Parnika Garg. 2020. Evaluation of Bacteriological Profile of Post Operative Wound Infections in Surgical Wards. Int.J.Curr.Microbiol.App.Sci. 9(02): 3224-3228. doi: https://doi.org/10.20546/ijcmas.2020.902.363 